\title{
Effect of kinematic viscosity on the staining performance of caries detector dyes
}

\author{
Mikihiro KOBAYASHI ${ }^{1}$, Rina INAGAKI', Kotona ICHIKAWA'1 , Yuiko NIIZUMA ${ }^{1}$, Hirobumi MORISAKI², \\ Hirotaka KUWATA2 ${ }^{2}$, Atsufumi MANABE ${ }^{1}$ and Takashi MIYAZAKI ${ }^{3}$
}

\author{
${ }^{1}$ Department of Conservative Dentistry, Division of Aesthetic Dentistry and Clinical Cariology, Showa University School of Dentistry, 1-1-2 Kitasenzoku, \\ Ohta-ku, Tokyo 145-8515, Japan \\ ${ }^{2}$ Department of Oral Microbiology and Immunology, Showa University School of Dentistry, 8-5-1 Hatanodai, Shinagawa-ku, Tokyo 142-8555, Japan \\ ${ }^{3}$ Department of Conservative Dentistry, Division of Oral Biomaterials and Technology, Showa University School of Dentistry, Hatanodai, Shinagawa- \\ ku, Tokyo 142-8555, Japan \\ Corresponding author, Mikihiro KOBAYASHI; E-mail: mkobayashi@dent.showa-u.ac.jp
}

\begin{abstract}
The relationship between performance of caries detector dyes and solvent molecular weight has been reported, but viscosity has not yet been investigated. Therefore, using extracted human teeth, we compared 6 experimental caries detector dyes with different viscosities to clarify the relationship between dye viscosity and staining performance in sclerotic dentin. Carious dentin started showing dentin staining when stained with caries detector dyes with kinematic viscosity $\geq 263 \mathrm{~mm}^{2} / \mathrm{s}$, showed no immunoreactivity for Streptococcus mutans on immunohistochemistry, and contained dentinal tubules free of debris on scanning electron microscopy. Samples stained using a caries detector dye with kinematic viscosity of $332 \mathrm{~mm}^{2} / \mathrm{s}$ contained calcification product-like debris in the dentinal tubules. These findings suggest that the caries detector dye has a threshold kinematic viscosity between 263 and $332 \mathrm{~mm} / \mathrm{s}$ at which sclerotic dentin and tissues beyond are not stained. The caries detector dye appears to control tooth dyeability based on solvent viscosity.
\end{abstract}

Keywords: Caries detection, Kinematic viscosity, Staining performance, Immunohistochemistry

\section{INTRODUCTION}

In recent years, the concept of minimal intervention (MI) has been promoted in the clinical management of dental caries $^{1-3)}$. First proposed by the Fédération Dentaire Internationale (FDI) in $2000^{1}$, the principle of MI is remineralization therapy for non-cavitated carious lesions and minimally invasive restoration for cavitated carious lesions ${ }^{4}$. Today, MI is not only a concept, but also a standard treatment approach owing to the establishment of the dentin-bonding system and advances in adhesive technology.

Carious dentin is broadly divided into two layers ${ }^{5,6}$ : the outer and inner layers. Previous studies have shown that even though the outer layer cannot be remineralized due to the destruction of collagen fibers, the inner layer retains the ability to undergo remineralization if treated properly ${ }^{7-9)}$, suggesting that only the outer layer should be removed during treatment. However, conventional approaches for caries removal are decided subjectively by dental surgeons based on their past experience, the hardness of carious tissue, and staining intensity ${ }^{10-13)}$. Against this background, a caries detector dye, a mixture of propylene glycol and Acid Red, was developed as an objective index of carious dental tissue for removal ${ }^{14-16)}$. As stated in the manufacturer's instructions ${ }^{14)}$, carious dentin is removed after applying this dye into a carious cavity and rinsing it off after a few seconds. However, there have been much debate on the removal of the stained border, that is, the area stained light pink. The current consensus is that dental tissue should not be removed when the tissue is stained light pink ${ }^{17)}$ because its removal would result in the loss of the dentin that is uninfected and has an ability to undergo remineralization ${ }^{9,18-20)}$. In addition, because the assessment of staining intensity is a subjective process, it goes against the purpose of using the caries detector dye ${ }^{21,22)}$. Consequently, propylene glycol, the solvent of the caries detector dye, was replaced with polypropylene glycol, which has chemical properties similar to propylene glycol but has lower permeation. Because this dye does not stain dental tissue beyond sclerotic dentin, by simply removing the area stained by the dye, it is possible to remove carious dentin completely and preserve the inner layer. The dye is commercially available under the name Caries Check ${ }^{\mathrm{TM}}$ (Nishika, Yamanashi, Japan) ${ }^{23-277}$.

As described above, the viscosity of the dental caries detector solution has been inferred to be correlated with the stainability of carious dentin. In previous research, the dyeability of carious dentin was controlled by increasing the viscosity via increases in the molecular weight of the solvent in order to avoid staining the inner layer of carious dentin (sclerotic dentin) which should not be removed. However, details of the relationship between viscosity and dyeability remain to be clarified. Therefore, in this study, the correlation between the viscosity of dental caries detector solution and stainability of carious dentin was examined and dental caries detector solutions with various viscosities were tested $^{23-277}$. In addition, we investigated whether the dyed portion corresponds to the outer layer of carious dentin, measured the hardness of the dentin surface after 
cutting and removal when using caries detector solution of various viscosities as an indicator, and checked for the presence of bacterial infection. The validity of the staining range was demonstrated.

The caries detector dye is currently being modified to reduce its permeation into dental tissue by replacing the solvent with one of a higher molecular weight ${ }^{28)}$. In general, fluid viscosity increases as the molecular weight of the solvent increases. Similarly, the viscosity of Caries Check ${ }^{\mathrm{TM}}$ detector dyes may be higher than that of caries detector dye with propylene glycol ${ }^{27)}$. Because dye viscosity affects staining performance in carious dentin, this physical property is an extremely important parameter. While the relationship between the performance of caries detector dyes in carious dentin and the molecular weight of the solvent has been reported ${ }^{27)}$, viscosity has not yet been investigated.

Accordingly, to prevent dyes from staining dental tissue beyond sclerotic dentin, this study analyzed the correlation between dye viscosity and staining performance using experimental caries detector dyes.

In addition, for bacteriological validation of the staining range, immunostaining was performed after removing dentin stained by the dye to reveal Streptococcus mutans remaining at the bottom of the carious cavity. Furthermore, the cross-section of the dentin was examined by scanning electron microscopy (SEM) to evaluate the surface features.

\section{MATERIALS AND METHODS}

\section{Extracted human teeth}

Dental specimens used in this study were 54 molars and premolars with mild dentine caries in the crown that were extracted from human adults. This study was approved by the Ethics Committee of Showa University School of Dentistry (approval No. 2014-037).

\section{Experimental caries detector dyes}

The components of Caries Check ${ }^{\mathrm{TM}}$ (Nishika) were used as base components. While keeping the dye concentration constant, the viscosity of the experimental dyes was adjusted with water (Table 1). The experimental caries detector dyes were prepared by diluting acid red in polypropylene glycol solution at a concentration of $1 \mathrm{wt} \%$. The dye with the most kinematic viscosity (R1) was prepared with the same kinematic viscosity as the commercially available Caries Check ${ }^{\mathrm{TM}}$ as control.

In accordance with the Japanese Pharmacopoeia, a glass capillary kinematic viscometer (Cannon-Fenske viscometer, size 350, Kusano Scientific, Tokyo, Japan) was used to measure kinematic viscosity.

\section{Dentin staining performance of caries detector dyes}

A flow chart of the experimental procedures is shown in Fig. 1. By polishing with silicon-carbide paper (\#100, \#250, and \#1000), the adjacent tooth surface was ground parallel to the conical bottom of the carious cavity, and thus parallel to the tooth axis. The dental specimens with carious dentin removed were subjected to hardness measurements using a Vickers micro-hardness tester (MMT-X3, Matsuzawa, Akita, Japan), which was set at 200 gf and $20 \mathrm{~s}$, and the DIAGNOdent ${ }^{\circledR}$ pen caries detection device (KaVo, Biberach, Germany) to prepare sclerotic dentin specimens. We cut a dental specimen until the hardness of the conical bottom of the caries was $35 \pm 5$ on the Vickers micro-hardness testing device and then determined the DIAGNOdent ${ }^{\circledR}$ (D) value to be around 20 at the cavity bottom, based on the above reference values. Dental caries satisfying both conditions were defined as inner layers and used as test specimens.

After removing carious tissue from 10 dental specimens created under these conditions, the inner dentin layer was stained for $3 \mathrm{~s}$ with different experimental caries detector dyes in the order of least viscous one (R1) to the most viscous one (R6). When no R1 staining was observed after rinsing with water, the same procedure was repeated to stain the sample with R2 and so on until the dentin was stained. Then, the corresponding caries detector dye and its kinematic viscosity were recorded. Dentin staining was evaluated macroscopically by three dentists with $\geq 4$ years of clinical experience.

\section{Immunohistochemical evaluation of dye performance}

Dental specimens were embedded in a chemically cured resin (Palapress ${ }^{\circledR}$ vario, Heraeus Kulzer, Hanau, Germany) and cut into $350-\mu \mathrm{m}$ sections parallel to the tooth axis using a diamond band saw (BS-312, EXAKT,

Table 1 Caries detection dyes and their kinematic viscosities

\begin{tabular}{lrrrrrr}
\hline \multicolumn{1}{c}{ Component } & R1 & R2 & R3 & R4 & R5 & R6 \\
\hline Polypropylene glycol (g) & 97 & 94 & 92 & 89 & 79 \\
Acid Red (g) & 1 & 1 & 1 & 1 & 1 \\
Water (g) & 2 & 5 & 7 & 10 & 15 & 20 \\
Viscosity (mm $/ \mathrm{s})$ & 461 & 377 & 332 & 263 & 157 \\
\hline
\end{tabular}

Polypropylene glycol: Polypropylene glycol with average molecular weight of 300 (Wako Pure Chemical Industries, Osaka, Japan), Acid Red: Acid Red 52 (Wako Pure Chemical Industries). Samples were prepared by mixing the components at the indicated composition ratios and stirring. 


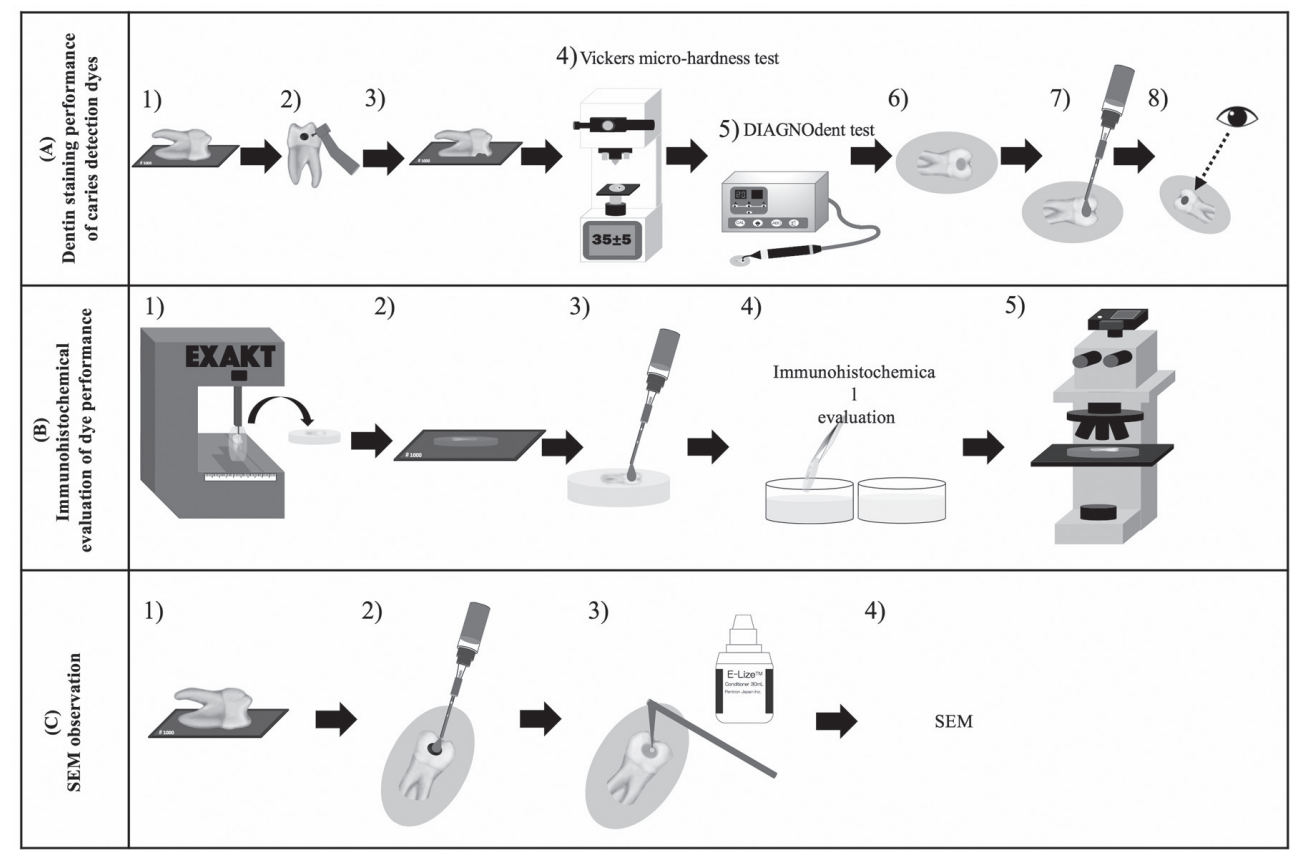

Fig. 1 Schematic flow chart of the experimental procedures.

A: Dentin staining performance of caries detector dyes.

1) Using a silicon-carbide paper (\#1000), the specimen was ground parallel to the adjacent tooth surface of the conical bottom of the carious cavity. 2) Caries were removed with steel burs (Gebr. Brasseler, Lemgo, Germany). 3) The specimen was polished with a silicon-carbide paper (\#1000). 4) The Vickers micro-hardness test (MMT-X3; Matsuzawa; 200 gf, 20 s) was conducted. We cut a dental specimen until the Vickers hardness of the conical bottom of caries was $35 \pm 5$. 5) We determined the $\mathrm{D}$ value to be around 20 at the cavity bottom, using the DIAGNOdent caries detection device (2095J; KaVo). 6) Only the carious dentin satisfying the conditions of 4) and 5) was used as a specimen. 7) The second dentin layer was stained for $3 \mathrm{~s}$ with individual experimental caries detector dyes in the order of R1 to R6. 8) The kinematic viscosity of the dental caries detector solution with which staining first occurred was confirmed.

B: Immunohistochemical evaluation of dye performance.

1) Carious dentin cut into $350-\mu \mathrm{m}$ sections parallel to the tooth axis using a diamond band saw. 2) Sections polished using silicon-carbide paper (\#1000). 3) Anti-WapA antibody, a surface protein of $S$. mutans, and anti-rabbit IgG antibody conjugated to alkaline phosphatase (Roche) were used as the primary and secondary antibodies. 4) Immunostaining of no dentin removal, $\mathrm{R} 1, \mathrm{R} 2, \mathrm{R} 3$, and $\mathrm{R} 4$ ( $n=4$ each) on upright microscopy.

C: SEM.

1) Using a silicon-carbide paper (\#1000), the specimen was ground parallel to the adjacent tooth surface of the conical bottom of the carious cavity. 2) Specimens were stained with R1 to R6. 3) The smear layer on the cavity wall was removed using $0.5 \mathrm{~mol} / \mathrm{L}$ neutralized EDTA ( $\mathrm{pH} 7.4$ ) for $60 \mathrm{~s}$ followed by rinsing and drying. 4) The microstructure of the surface and a section of cavity wall were observed by SEM (S-4700, Hitachi; 5.0 KV) after vacuum deposition of palladium and platinum.

Tokyo, Japan). Then, the sections were polished using silicon-carbide paper (\#1000). Anti-WapA antibody, a surface protein of $S$. mutans, and anti-rabbit IgG antibody conjugated to alkaline phosphatase (Roche Diagnostics, Tokyo, Japan) were used as the primary and secondary antibodies. Colorimetric assay was performed using an NBT/BCIP stock solution (Roche), and immunostaining of no dentin removal, R1, R2, R3, and R4 ( $n=4$ each) was observed using an upright microscope (Nikon, Tokyo, Japan).

Scanning electron microscopy observation of dentin after removal of the stained area

The surface features of dentin in specimens stained by R1 to R6 were examined by using SEM (S-4700, Hitachi, Tokyo, Japan; pressurized voltage, $5.0 \mathrm{kV}$ ) after vapor deposition of gold-palladium onto the surface of the tooth $(n=4$ each). 


\section{RESULTS}

Dentin staining performance of caries detector dyes with different kinematic viscosities

Table 2 shows the results of staining starting from R1. Of the 10 samples, 6 were stained by $\mathrm{R} 4$ and the remaining 4 were stained by R5 (Fig. 2).

In addition, no significant difference in microhardness $(34.4 \pm 4)$ and DIAGNOdent ${ }^{\circledR}$ values $(16.7 \pm 3)$ was observed in the areas stained by R4 and R5 (Table 3).

Immunohistochemical evaluation of dye performance Figure 3 shows the outcome of immunostaining performed using five consecutive sections. Although the section in Fig. 3A shows heavy staining for S. mutans, the three sections in Figs. 3B, C, and D showed only very light staining for $S$. mutans, with no clear difference among the three sections. No staining was observed in the section in Fig. 3E.

\section{SEM observation of dentin after removal of the stained area}

Figure 4 shows the results of SEM. Calcification product-like debris was observed in the dentinal tubules in all four samples stained by R1 to R3 (Figs. 4A, B, C), but not in those stained by R4 to R6 (Figs. 4D, E, F). In the latter, the tubules did not appear to be blocked by any debris.

Table 2 Number of dental specimens stained by caries detection dyes $(n=10)$

\begin{tabular}{cccccc}
\hline & R1 & R2 & R3 & R4 & R5 \\
\hline Stained samples (n) & 0 & 0 & 0 & 6 & 4 \\
\hline
\end{tabular}
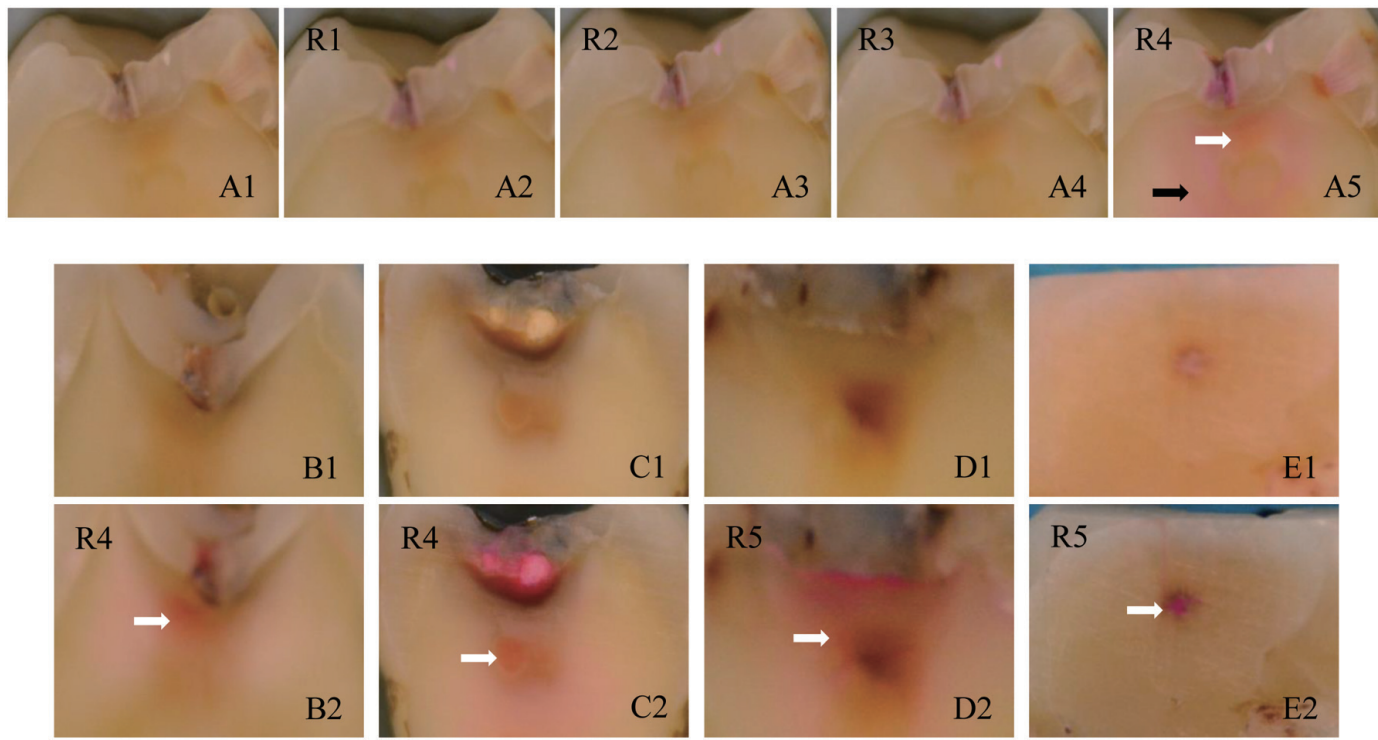

Fig. 2 Affinity of individual caries detector dyes toward the adjusted dentin samples.

The cavity after removal of carious dentin (A1, B1, C1, D1, E1). Kinematic viscosity of the prototype caries detector solution for which dyeing was confirmed at the outset (A5, B2, C2, D2, E2); the stained sclerotic dentin is also shown (white arrow). Dentin not affected by dental caries was stained (black arrow).

Table 3 Vickers micro-hardness and DIAGNOdent value (D-value) measurement results

\begin{tabular}{ccc}
\hline & Micro vickers hardness & D-value \\
\hline R4 & $34.7 \pm 3.2^{\mathrm{a}}$ & $17.3 \pm 3.2^{\mathrm{b}}$ \\
R5 & $34.0 \pm 4.5^{\mathrm{a}}$ & $16.3 \pm 3.3^{\mathrm{b}}$ \\
\hline
\end{tabular}

Data are shown as the mean \pm standard deviation $(n=10)$. Identical letters indicate no statistically significant difference $(p>0.05, t$-test). 


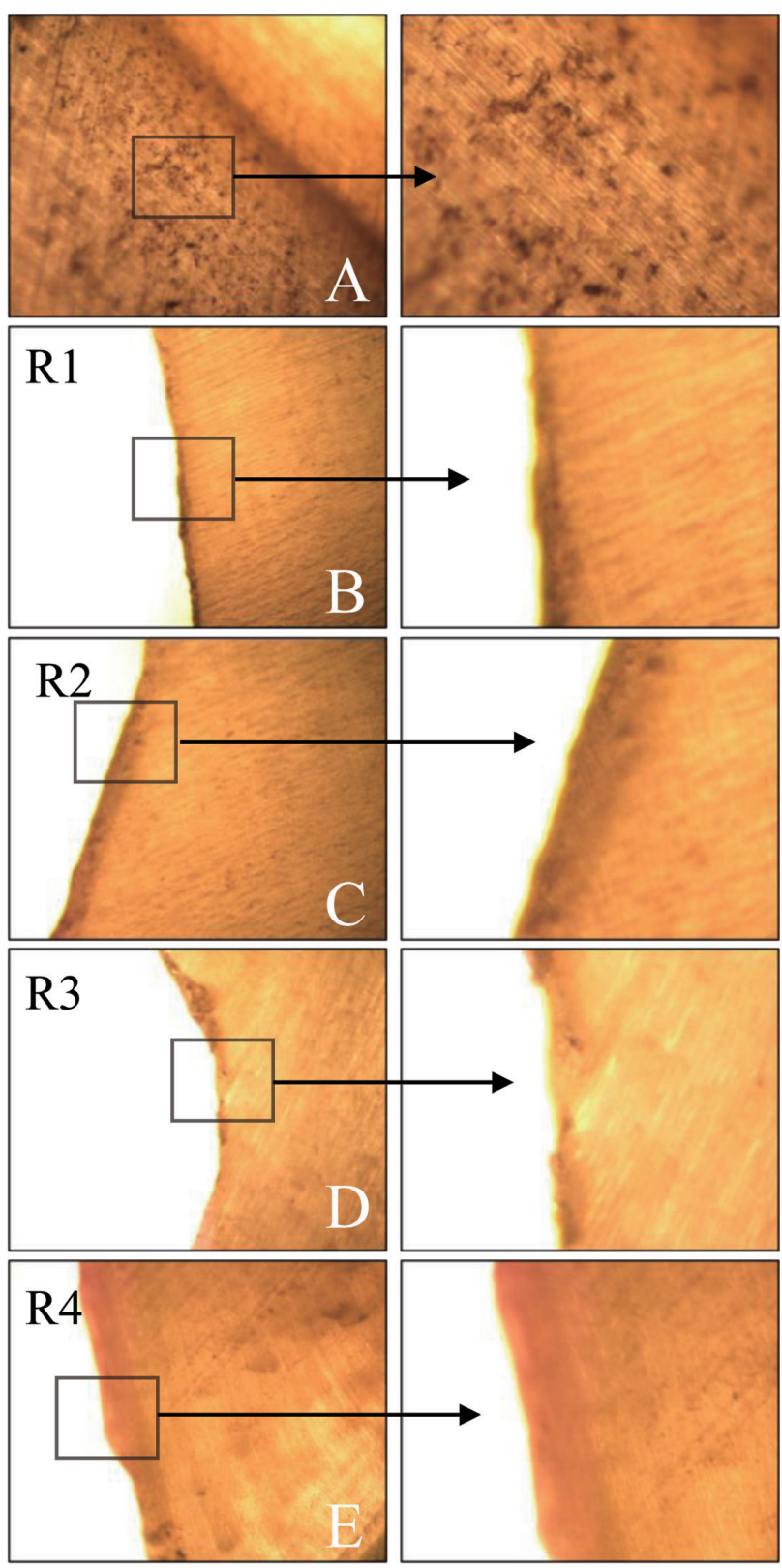

Fig. 3 Immunostained S. mutans.

A: No dentin removal. B: Dentin removal with round bur after staining dentin caries with R1. C: Dentin removal with round bur after staining dentin caries with R2. D: Dentin removal with round bur after staining dentin caries with R3. E: Dentin removal with round bur after staining dentin caries with R4. A large quantity of $S$. mutans was observed in image $\mathrm{A}$. In images $\mathrm{B}, \mathrm{C}$, and $\mathrm{D}$, a trace amount of $S$. mutans was observed but was negligible. In image E, no $S$. mutans was observed.

\section{DISCUSSION}

After the pioneering work by Fusayama et al., the utility of caries detector dyes as indicators of carious dentin removal has been intensively investigated ${ }^{18-20)}$.

In prior research, a caries detector dye was developed by mixing propylene glycol with acid red ${ }^{16,17)}$, but the dentin stained light pink by the dye should be preserved because it is free from bacterial infection and is capable of undergoing remineralization ${ }^{17)}$. However, the assessment of the light pink staining by dental surgeons is a subjective process; therefore, a caries detector dye (Caries Check ${ }^{\mathrm{TM}}$, Nishika) was developed by replacing propylene glycol with polypropylene glycol, which has a higher molecular weight ${ }^{23-27)}$, because fluid viscosity generally increases as the molecular weight of the solvent increases. In addition, dye viscosity greatly affects staining performance in carious dentin ${ }^{21)}$. Indeed, Caries Check $^{\mathrm{TM}}$ does not stain dental tissue beyond sclerotic dentin because of the higher molecular weight of the solvent ${ }^{23-27)}$. Although previous studies showed an association between the performance of caries detector dyes on carious dentin and the molecular weight of the solvent ${ }^{27)}$, no study has investigated the association between dye performance and the amount of water, that is, fluid viscosity.

In this study, we evaluated the performance of experimental caries detector dyes to clarify the relationship between dye viscosity and the staining performance necessary to prevent the dye from staining dental tissue beyond sclerotic dentin.

Caries detector dyes with different kinematic viscosities In a previous study using a caries detector dye and extracted molars with moderate occlusal caries, Oikawa et al. reported that the Vickers micro-hardness of the dentin in the cavity wall was $34.7 \pm 5.5$ after thoroughly removing the stained dentin ${ }^{27)}$.

In addition, Lussi et al. showed that the DIAGNOdent values (D values) after removing the area stained by the caries detector dye was 0-13 for unnecessary treatment (healthy dentin), 14-20 for preventive treatment and follow-up observation, and $\geq 21$ for necessary treatment (corresponding to the infected dentin layer ${ }^{29,30)}$. When staining was performed, the sclerotic dentin samples were stained by $\mathrm{R} 4$ with a kinematic viscosity of $263 \mathrm{~mm}^{2} / \mathrm{s}(n=6)$ and R5 with a kinematic viscosity of $157 \mathrm{~mm}^{2} / \mathrm{s} \quad(n=4)$. This suggests that it was easier for these dyes to infiltrate and spread through the dentin because of the higher water content and lower viscosity compared with the other dyes. These findings also suggest that caries detector dyes should have water content and viscosity comparable with those of R3 (water content, 7\%; kinematic viscosity, $332 \mathrm{~mm}^{2} / \mathrm{s}$ ), but not those of R4 (water content, 10\%; kinematic viscosity, $263 \mathrm{~mm}^{2} / \mathrm{s}$ ) or R5 (water content, $20 \%$; kinematic viscosity, $103 \mathrm{~mm}^{2} / \mathrm{s}$ ) because of the increased water content and reduced kinematic viscosity in the latter two.

In addition, to bacteriologically investigate the validity of the relationship between dye viscosity and performance on carious dentin, immunostaining was performed to detect $S$. mutans remaining at the bottom of the dentin cavity after the removal of dentin 

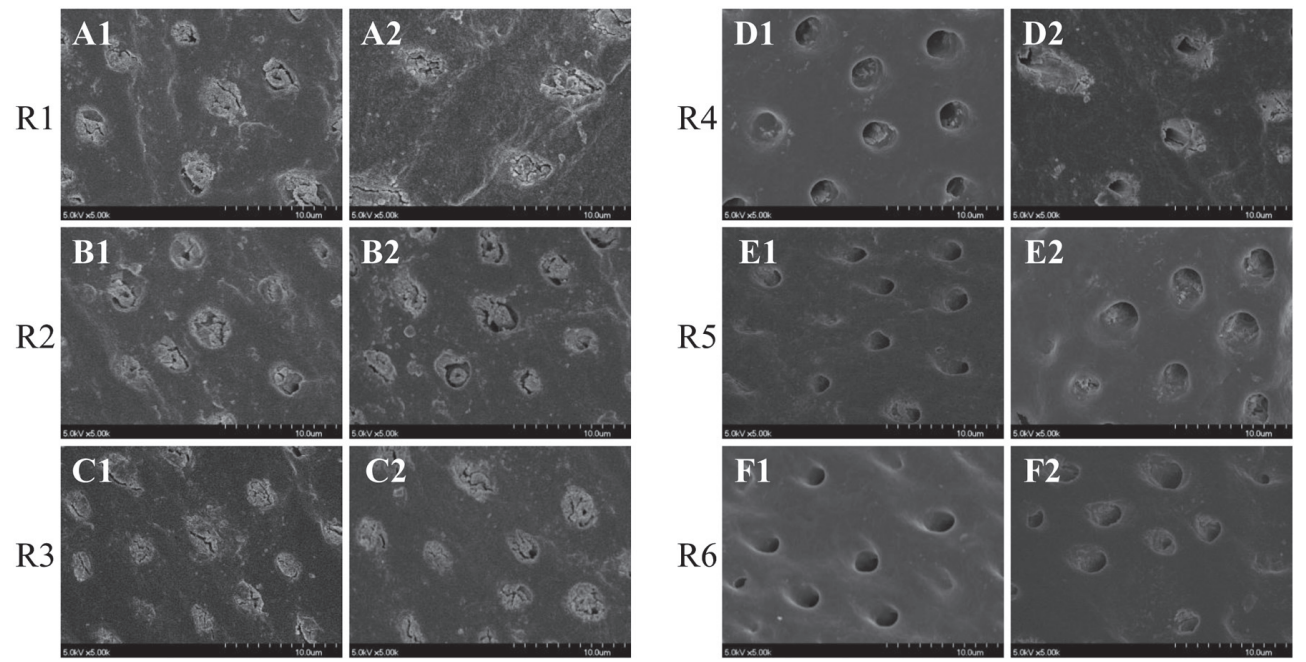

Fig. 4 SEM image showing dentinal tubules (5,000× magnification).

A: Dentin removal with round bur after staining dentin caries with R1. B: Dentin removal with round bur after staining dentin caries with R2. C: Dentin removal with round bur after staining dentin caries with R3. D: Dentin removal with round bur after staining dentin caries with R4. E: Dentin removal with round bur after staining dentin caries with R5. F: Dentin removal with round bur after staining dentin caries with R6. The dentin tubules of A1, A2, B1, B2, C1, and C2 were filled with debris. A few dentin tubules of D1, E1 were filled with debris. The dentin tubules of D2, E2, F1, and F2 were completely empty.

stained by the dye. The mutans streptococci, which are composed of seven species including S. mutans, are considered the most important cariogenic bacteria. Among the seven species, S. sobrinus and especially $S$. mutans have been studies intensely to elucidate their involvement in human caries $^{31,32)}$. Therefore, we used $S$. mutans in this study. Despite the clear presence of $S$. mutans after the removal of carious dentin stained by R1-R3, no bacterial staining was observed in the sample stained by R4. Compared with the sample stained but did not undergo the removal of stained tissue (Fig. 3A), the samples stained by R1 (Fig. 3B), R2 (Fig. 3C), or R3 (Fig. 3D) showed very weak immunostaining for $S$. mutans after the removal of stained dental tissue, with no significant difference among R1-R3. In addition, the sample stained by R4 showed no staining for $S$. mutans after the removal of stained tissue (Fig. 3E). It has been reported that bacteria were still detected in some teeth after complete removal of carious dentin with the aid of caries detector dye ${ }^{33,34)}$. For immunostaining, we use an antibody against proteins present on the cell surface of S. mutans. This does not depend on whether the bacteria are alive or dead. In our study, only a trace number of bacteria was detected, and so infected dentin is considered to have almost completely disappeared.

SEM of dentin cross-sections after carious dentin removal revealed calcification product-like debris in the R3 samples, whereas the dentinal tubules free of debris were observed in the R4 samples. A previous study showed the sedimentation of microcrystalline tricalcium phosphate complex in the dentinal tubules after the removal of carious dentin, whereas the dentin tubules were free of any sedimentation after the excessive removal of carious dentin ${ }^{32}$. The calcification productlike debris observed in the dentinal tubules in the R3 samples after the removal of stained tissue appeared to be microcrystalline calcium phosphate complex, suggesting that no excessive removal of dentin was occurred in the sample. Such dentin should not be removed because of its ability to undergo remineralization ${ }^{35-37)}$. Our data also suggest that the dentin was removed excessively in the R4 samples.

From these results, we conclude that in the R3 samples, sclerotic dentin was preserved after the removal of stained tissue, whereas even sclerotic dentin was removed in the R4 samples. In sum, dentin needed for remineralization was unnecessarily removed in the sample stained by $\mathrm{R} 4$.

\section{CONCLUSION}

Investigating the correlation between the viscosity of the solvent and the dyeability of the carious dentin confirmed that viscosity directly affects the degree of dyeing and, established the critical threshold viscosity.

This study suggests that the caries detector dye has a threshold kinematic viscosity between 263 and 332 $\mathrm{mm}^{2} / \mathrm{s}$, at which sclerotic dentin and tissues beyond are not stained. 


\section{CONFLICTS OF INTEREST}

We have no conflict of interest to report.

\section{REFERENCES}

1) Tyas MJ, Anusavice KJ, Frencken FE, Mount GJ. Minimal intervention dentistry - a review. FDI Commission Project 1-97. Int Dent J 2000; 50: 1-12.

2) Murdoch-Kinch CA, McLean ME. Minimally invasive dentistry. J Am Dent Assoc 2003; 134: 87-95.

3) Mount GJ, Ngo H. Minimal intervention; a new concept for operative dentistry. Quintessence Int 2000; 31: 527-533.

4) FDI policy statement: minimal intervention in the management of dental caries; adopted by the FDI General Assembly: 1 October 2002-Vienna.Vienna: FDI; 2002.

5) Fusayama T, Terashima S. Differentiation of two layers of carious dentin by staining. J Dent Res 1972; 51: 866.

6) Fusayama T. Two layers of carious dentin; diagnosis and treatment. Oper Dent 1979; 4: 63-70.

7) Ohgushi K, Fusayama T. Electron microscopic structure of the two layers of carious dentin. J Dent Res 1975; 54: 10191026.

8) Kuboki Y, Ohgushi K, Fusayama T. Collagen biochemistry of two layers of carious dentin. J Dent Res 1977; 56: 1233-1237.

9) Miyauchi, H, Iwaku, M, Fusayama, T. Physiological recalcification of carious dentin. Bull Tokyo Med Dent Univ 1978; 25: 169-179.

10) Banerjee A, Watson TF, Kidd EA. Dentine caries: take it or leave it? Dent Update 2000; 27: 272-276.

11) Kidd E, Toyston-Bechal S, Beighton D. Microbiological validation of assessments of caries activity during cavity preparation. Caries Res 1993; 27: 402-408.

12) Weerheijm KL, de Soet JJ, van Amirongen WE, de Graaff J. The effect of grass-ionomer cement on caries dentin: an in vivo study. Caries Res 1993; 27: 417-423.

13) Kidd EA, Fejerskov O, Mjor I. Caries removal and the pulpodentinal complex. In: Fejerskov O, Kidd E, editors. Dental caries: the disease and its clinical management. 1st ed. Oxford: Blackwell Munksgaard; 2003. p. 267-274.

14) Fusayama T. Clinical guide removing caries using a cariesdetecting solution. Quintessence Int 1988; 19: 397-401.

15) Zacharia MA, Munshi AK. Microbiological assessment of dentin stained with a caries detector dye. J Clin Pediatr Dent 1995; 19: 111-115.

16) Itoh $\mathrm{K}$, Kusunoki M, Oikawa M, Tani C, Hisamitsu H. In vitro comparison of three caries dyes. Am J Dent 2009; 22: 195-199.

17) Inokoshi S. Caries detection dyes that do not overstain. Dental Outlook 2005; 106: 303-304 (in Japanese).

18) Fukushima M. Investigation of infiltration of adhesive resin into carious dentin. J Stomatol Soc Jpn 1981; 48: 362-335 (in Japanese).

19) ten Cate JM. Remineralization of caries lesions extending into dentin. J Dent Res 2001; 80: 1407-1411.

20) Inokoshi S. Use of a caries detection dye to remove infected dentin. Dental Outlook 1997; 90: 328-335 (in Japanese).

21) Shimizu A, Maeda T, Natsume Y, Hasegawa M, Honda K. Development and evaluation of a prototype device to measure the hardness of carious dentin. Jpn J Conserv Dent 2004; 47: 469-475 (in Japanese).

22) Commission International de l'Eclairage. CIE technical report colorimetry. 2nd ed. Vienna: CIE Central Bureau; 1986. p. 3031.

23) Oikawa M, Kusunoki M, Itoh K, Hisamitu H. The experimental caries detector containing polypropylene glycol. J Dent Res 2004; 83: A0757.

24) Ishizaki Y, Fukushima M. Novel handy device useful in clinical practice. Dental Diamond 2004; 29-16: 146-149 (in Japanese).

25) Nishikawa Y. A case of caries removal using Caries Check. Dental Outlook 2004; 104: 924-933 (in Japanese).

26) Tanaka J. Nishika Caries Check: a caries detection dye for the era of minimal intervention. Nippon Dental Review 2005; 65: 111-117 (in Japanese).

27) Oikawa M, Kusunoki K, Itoh K, Hisamitsu H. An experimental carious detector to stain the carious infected dentin. Dent Med Res 2008; 28: 7-12

28) Ito K. Establishment of a theory of dentin bonding through the application of EDTA and GM and the novel caries detection dye Caries Check. Dental Outlook 2004; 104: 910923 (in Japanese).

29) Lussi A, Megert B, Longbottom C, Reich E, Francescut P. Clinical performance of a laser fluorescence device for detection of occlusal caries lesions. Eur J Oral Sci 2001; 109: 14-19.

30) Tani C, Itoh K, Hisamitsu H, Wakumoto S. Efficacy of the dentin bonding to cervical defects. Dent Mater J 2001; 20: 359-368.

31) Axel P. Diagnosis and risk prediction of dental caries. Illinois: Quintessence; 2000. p. 18-19.

32) Oikawa M, Itoh K, Kusunoki M, Kitahara N, Miyazaki T. Efficacy of three caries-staining agents. Dent Med Res 2013; 33: 79 .

33) Lula EC, Mokonteriro-Neto V, Alves CM, Ribeiro CC. Microbiological analysis after complete or partial removal of carious dentin in primary teeth: a randomized clinical trial. Caries Res 2009; 43: 354-358.

34) Boston DW, Graver HT. Histological study of acid red cariesdisclosing dye. Oper Dent 1989; 14: 186-192.

35) Kusunoki M, Itoh K, Hisamitu H, Wakumoto S. The efficacy of dentin adhesive to scleratic dentin. J Dent 2002; 30: 9197.

36) Wu J, Itoh K, Yamashita T, Hisamitsu H, Wakumoto S. The efficacy of the dentin adhesive to the caries-affected dentin. J Showa Univ Dent Soc 2003; 23: 166-174.

37) Yip HK, Stevenson AG, Beeley JA. The specificity of caries detector dyes in cavity preparation. Br Dent J 1994; 176: 417421. 